\title{
Pneumatically tunable optofluidic dye laser
}

\author{
Wuzhou Song a) and Demetri Psaltis \\ School of Engineering, Swiss Federal Institute of Technology Lausanne (EPFL), CH-1015 Lausanne, \\ Switzerland
}

(Received 3 November 2009; accepted 5 January 2010; published online 22 February 2010)

\begin{abstract}
We presented a tunable optofluidic dye laser with integrated elastomeric air-gap etalon controlled by air pressure. The chip was fabricated with polydimethylsiloxane (PDMS) via replica molding. It comprises a liquid waveguide and microscale air-gap mirrors providing the feedback. The lasing wavelength is chosen by the interference between two parallel PDMS-air interfaces inside the internal tunable air-gap etalon, of which pneumatic tuning can be realized by inflating the air-gap etalon with compressed air. This dye laser exhibits a pumping threshold of $1.6 \mu \mathrm{J} / \mathrm{pulse}$, a lasing linewidth of $3 \mathrm{~nm}$, and a tuning range of $14 \mathrm{~nm}$. (c) 2010 American Institute of Physics.
\end{abstract}

[doi:10.1063/1.3324885]

Optofluidics has shown large potential for optical devices and has already enabled a broad optical toolbox for lab-on-a-chip (LOC) systems. ${ }^{1-4}$ As a miniature coherent light source, optofluidic dye lasers attract a lot of attention. Various types of resonators for microfluidic dye lasers have been demonstrated, such as the Fabry-Perot cavity, ${ }^{5}$ a capillary tube, ${ }^{6}$ and distributed feedback (DFB) grating. ${ }^{7-10} \mathrm{~A}$ common method for tuning the wavelength of such microfluidic dye lasers is based on varying the refractive indices of dye solutions. ${ }^{9,10}$ Although this method is simple, it has several shortcomings. First, the tuning range is very limited, especially for liquid core DFB dye lasers, ${ }^{8,9}$ since the refractive index distribution must also satisfy the single mode condition of the waveguide. As a result, the refractive index of the dye solution can be changed only in a very small range. Second, due to the small transverse dimension of the liquid waveguide, the flow inside the microfluidic channel is very slow; hence the waveguide tuning timing is long. Another tuning mechanism for optofluidic DFB dye laser was reported by $\mathrm{Li}$ et al. ${ }^{11}$ It is based on mechanical stretching or compressing a laser chip made of soft material [Polydimethylsiloxane (PDMS)], hence changing the period of the DFB grating to tune the lasing wavelength. However, the induced overall deformation of the chip would be at odds with the integration of such dye laser into more complex microfluidic system.

In this letter, we present an optofluidic laser cavity structure in which the lasing wavelength is chosen by an integrated pneumatic tuning air-gap etalon. As the etalon is made of PDMS which is a kind of elastomer, the air-gap etalon can be inflated by compressed air and the lasing wavelength can be controlled by the air pressure. Compared with previous tuning method, ${ }^{9-11}$ this implementation is simpler and the dye laser wavelength can be remotely controlled. Fast laser tuning and a large tuning range can be realized. Meanwhile, since the deformation is localized in the etalon, the tuning process does not interfere with the other components on the chip.

The schematic of the laser cavity structure is illustrated in Fig. 1. The whole chip is made of PDMS. It is composed of a liquid core waveguide, at the ends of which two micro-

${ }^{\text {a)}}$ Electronic mail: wuzhou.song@epfl.ch. scale air chamber structures are integrated acting as the mirrors providing feedback. The beam reflections occur at the PDMS/air interfaces due the refractive index contrast. As shown in Fig. 1(a), the shape of the air chamber located on the left, was specially designed. Only the interface closer to the liquid waveguide is effective for the cavity as the reflected beam can couple back to the waveguide. The other interface has a tilted mirror, from which the reflected beam does not couple back to the waveguide. As a result, the airgap mirror on the left acts as a simple end mirror for the laser cavity. On the other hand, the air chamber on the right of the waveguide, as shown in Fig. 1(b), has two parallel PDMS/air interfaces that are both perpendicular to the waveguide. Owning to interference of the reflected beams between the two interfaces, this air chamber behaves as a low finesse air-gap Fabry-Perot (F-P) etalon. This cavity determines the lasing wavelength. Since the PDMS is elastic, the size of the air-gap can be tuned by varying the gas pressure. ${ }^{12}$ As shown in the schematic cross-sectional view of Fig. 1(c) of the devices, inflation of the "microairbag" with compressed air causes the effective air-gap to increase.

To further illustrate the working principle of this laser, a model of F-P cavity laser with external mirror is presented in Fig. $2{ }^{13,14} F_{1}, F_{2}$, and $F_{3}$ denote the two facets of the F-P laser and the external mirror, $r_{1}$ and $r_{2}$ are the real values representing the amplitude reflectance of $F_{1}$ and $F_{2}$, respectively, while $r_{3}$ denotes the effective amplitude reflectance of

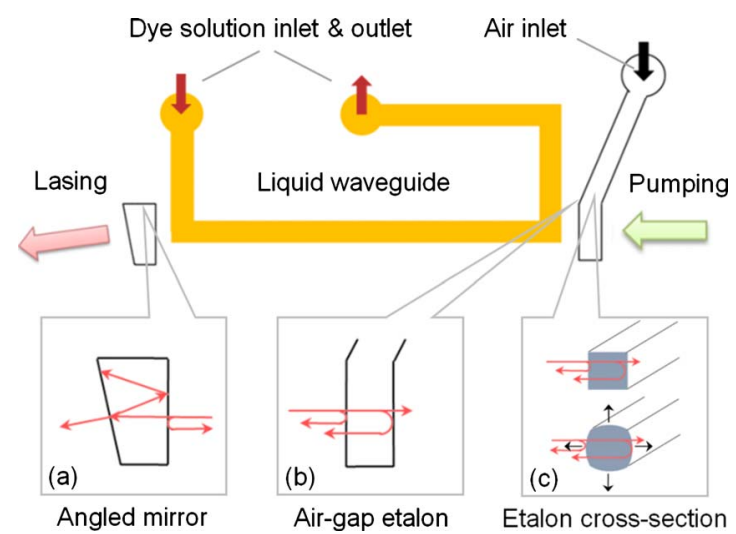

FIG. 1. (Color online) The schematic of the dye laser cavity structure. 


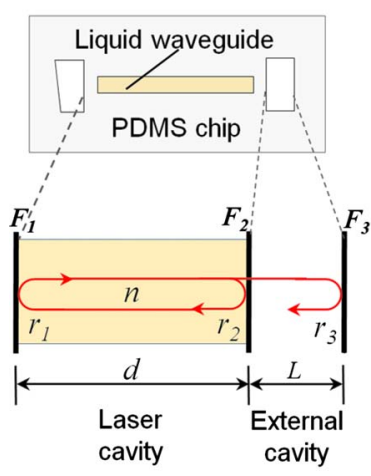

(a)

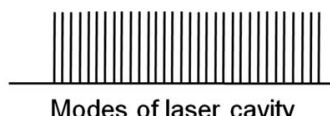

Modes of laser cavity

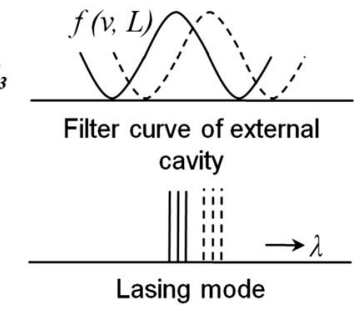

(b)
FIG. 2. (Color online) The schematic of the laser model based on F-P cavity laser with external mirror.

the external mirror $F_{3} . n$ is the effective refractive index of the lasing cavity. The length of the lasing cavity and the external cavity are $d$ and $L$, respectively. Due to the existence of the external mirror $F_{3}$, the effective reflectance $r_{2 \text { eff }}$ of the mirror $F_{2}$ is given by the following: ${ }^{14}$

$$
r_{2 \mathrm{eff}}=r_{2}-\frac{1-r_{2}^{2}}{r_{2}} \sum_{k=1}^{\infty}\left[r_{2} r_{3} \exp \left(-\frac{j 4 \pi L}{\lambda}\right)\right]^{k},
$$

where $\lambda$ is the wavelength in vacuum. Assuming the divergence of the beam reflected from mirror $F_{3}$ can be negligible, $r=r_{1}=r_{2}=r_{3} \approx 0.03$ using the refractive index of PDMS $n_{\text {PDMS }}=1.412$. Due to the weak reflection coefficient, higher orders can be negligible. $\left(r^{2}=0.0009 \ll 1\right), r_{2 \text { eff }}$ can be expressed as follows:

$$
\begin{aligned}
& r_{2 \mathrm{eff}}=R_{2} \exp \left(j \phi_{L}\right), \\
& R_{2}=r \sqrt{2\left[1-\cos \left(\frac{4 \pi L}{\lambda}\right)\right],} \\
& \phi_{L}=-\arcsin \left[\sin \left(\frac{4 \pi L}{\lambda}\right) / R_{2}\right],
\end{aligned}
$$

where $R_{2}$ and $\phi_{L}$ represent the amplitude and phase of $r_{2 \text { eff }}$. To obtain lasing, the following amplitude and phase condition must be simultaneity satisfied:

$$
\begin{aligned}
& r_{1} R_{2} \exp [(g-\gamma) d] \\
& \quad=r^{2} \exp [(g-\gamma) d] \sqrt{2\left[1-\cos \left(\frac{4 \pi L}{\lambda}\right)\right]}=1 \\
& \phi_{L}+\frac{4 \pi n d}{\lambda}=2 \pi m \quad(m=\text { integer }),
\end{aligned}
$$

where $g$ and $\gamma$ represent the gain and loss of the cavity respectively. The influence of the external cavity can be observed from Eqs. (5) and (6). In the absence of the external cavity $\left(R_{2}=r\right.$ and $\left.\phi_{L}=0\right)$, the F-P laser oscillation is fully determined by its gain profile and longitude modes. The presence of the external cavity alters both the gain condition and the longitudinal mode wavelength positions. However, as $\phi_{L} \ll 2 \pi m$, the mode positions of the laser cavity are almost as same as without external cavity. Therefore the external cavity mainly impacts the gain condition [Eq. (5)] which is equivalent to adding a tunable etalon inside the laser cav-

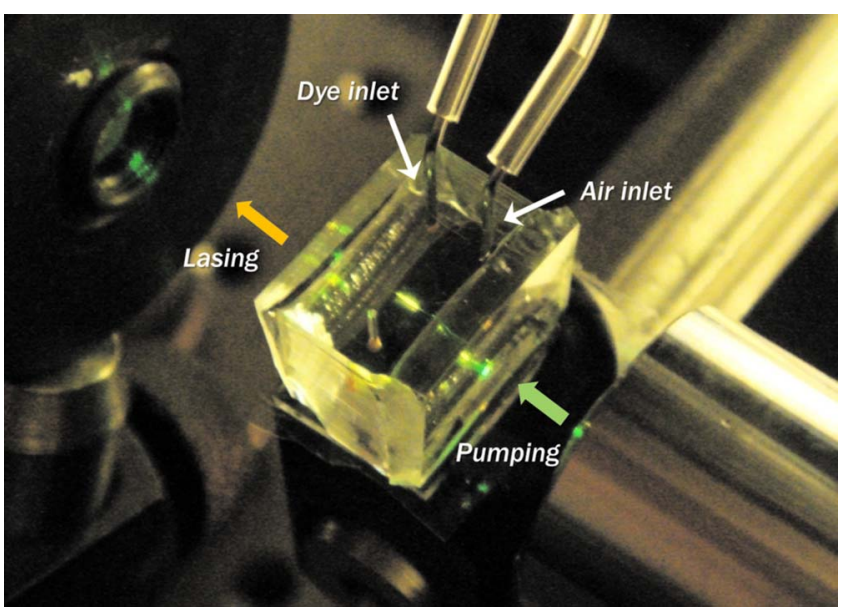

FIG. 3. (Color online) The picture of dye laser chip under testing.

ity. As illustrated in Fig. 2(b), the laser cavity modes are selected by the center wavelength of the filtering curve formed by the reflectance spectrum of the air-gap etalon.

The top PDMS layer of the chip was prepared by replica molding method. The master mold was defined using UV lithography on a $4 \mu \mathrm{m}$ thick SU-8 film on silicon wafer. The length of the waveguide is $800 \mu \mathrm{m}$ and the width $4 \mu \mathrm{m}$. The air chamber on the right acting as the external cavity has a gap width of $12 \mu \mathrm{m}$. The structured PDMS slab (Dow Corning Sylgard 184) was then bonded with a flat PDMS layer. A mixture of two different dyes was utilized to improve the tuning range. The chip was filled with a mixed dye solution containing rhodamine $6 \mathrm{G}$ and rhodamine $\mathrm{B}$ at the concentration of 0.9 and $0.15 \mathrm{mg} / \mathrm{ml}$, respectively. The solvent was prepared by mixing water and dimethylsulfoxide and had a refractive index of 1.4140. Figure 3 is the picture of a dye laser chip under testing. To improve the pumping efficiency, a longitude pumping scheme was adopted. ${ }^{8}$ The air inlet of the chip was connected to a mechanical air pressure regulator (ControlAir Inc., Type 100-BA) providing arbitrary pressure in the range of 2-30 psi. The pressure was monitored by a digital pressure gauge (Omega Inc. DPG4000).

A comparison of the emission spectra from the dye laser is shown in Fig. 4. The black line denotes a typical lasing

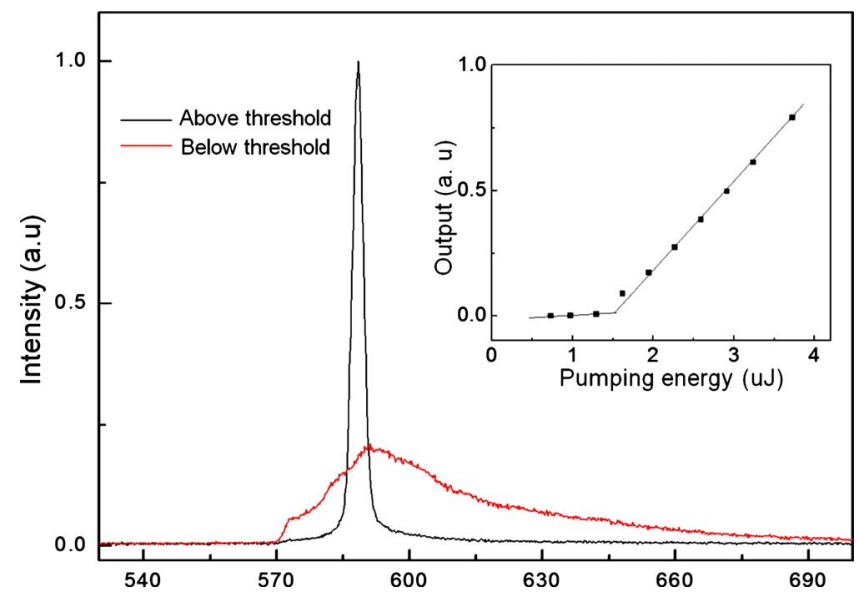

FIG. 4. (Color online) The caparison of the emission spectrum below and above lasing threshold. The inset shows the plots of output vs pumping energy. 


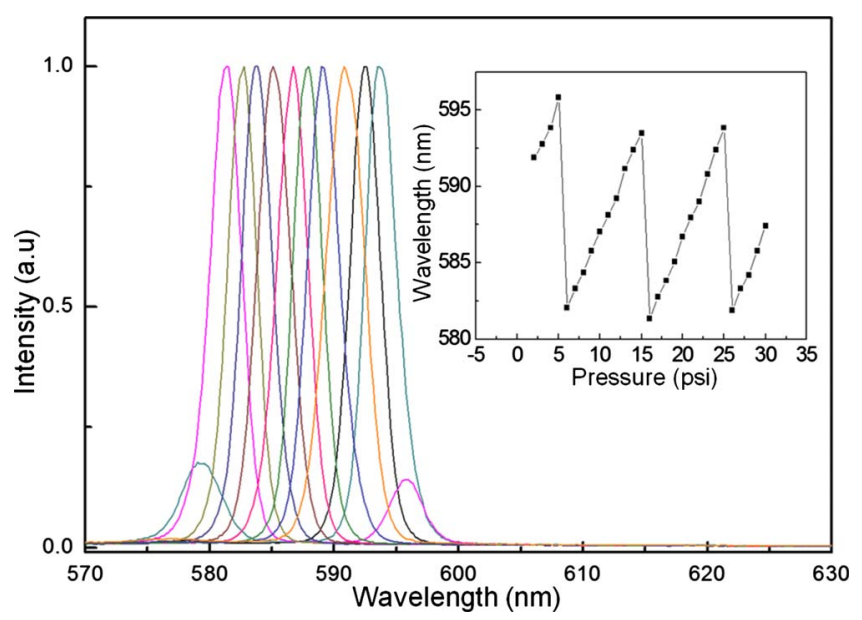

FIG. 5. (Color online) The superposition of the lasing spectrum in whole tuning range. The inset shows the relationship of lasing wavelength vs air pressure. (enhanced online). [URL: http://dx.doi.org/10.1063/1.3324885.1]

spectrum above the pumping threshold, and the red shows the emission spectrum bellow the lasing threshold. The laser emission appears at the wavelength of $588.4 \mathrm{~nm}$ with a linewidth of about $3 \mathrm{~nm}$. By calculating the free spectral range (FSR) of the liquid waveguide cavity, this lasing emission was expected to contain about 19 longitudinal laser modes. The plot of pumping pulse energy versus output pulse energy is shown in the inset of Fig. 4. The threshold pump energy was measured to be $1.6 \mu \mathrm{J} / \mathrm{pulse}$. The dye laser tuning was controlled by air pressure. As shown in the superposition graph of the laser emission spectra in Fig. 5, a tuning range of $14 \mathrm{~nm}$ was realized which agrees with the estimated FSR of the etalon. The inset of Fig. 5 shows the relationship of the lasing wavelength versus air pressure. As limited by FSR of the etalon and also the dye solution's gain profile, the laser tuning shows clear cycle behavior with the air pressure.

There are two following contributing factors to the wavelength tuning: the geometrical variation of the etalon cavity and the refractive index change of the air inside the cavity. From the calculation, during the increment of air pressure, the cavity length has an increment rate of $33 \mathrm{~nm} / \mathrm{psi}$ and air refractive index has an increment rate of 2 $\times 10^{-5} / \mathrm{psi}$, in which the cavity geometric modification can be considered as the primary factor for the wavelength tuning. Due to the low viscosity, the air flow has a much higher circulating rate inside the microchannels compared to the liquid. Thus such pneumatic tuning can exhibit much faster response time compared to the tuning methods using liquid flow. ${ }^{8-10}$ In addition, thanks to the separate air-gap etalon for selecting the lasing wavelength, this dye laser shows nice wavelength stability compared to the liquid-core waveguide DFB dye laser, in which the variation of refractive index induced by thermal fluctuation can shift the lasing wavelength. ${ }^{8}$ In contrast, this dye laser chip showed negligible wavelength variation by locking the pressure with the mechanical air regulator.

In conclusion, we demonstrated a pneumatically tunable optofluidic dye laser with integrated elastomeric air-gap etalon. As the fabrication process and control method are compatible with conventional a PDMS based microfluidic chip, ${ }^{15}$ the design of this optofluidic dye also promises a practical way for the integration of dye laser source into lab-on-a-chip system.

${ }^{1}$ D. Psaltis, S. R. Quake, and C. Yang, Nature (London) 442, 381 (2006). ${ }^{2}$ C. Monat, P. Domachuk, and B. J. Eggleton, Nat. Photonics 1, 106 (2007).

${ }^{3}$ W. Z. Song, X. M. Zhang, A. Q. Liu, C. S. Lim, P. H. Yap, and H. M. M. Hosseini, Appl. Phys. Lett. 89, 203901 (2006).

${ }^{4}$ W. Z. Song, A. Q. Liu, S. Swaminathan, C. S. Lim, P. H. Yap, and T. C. Ayi, Appl. Phys. Lett. 91, 223902 (2007).

${ }^{5}$ Q. Kou, I. Yesilyurt, and Y. Chen, Appl. Phys. Lett. 88, 091101 (2006).

${ }^{6}$ X. Wu, Y. Sun, J. D. Suter, and X. Fan, Appl. Phys. Lett. 94, 241109 (2009).

${ }^{7}$ W. Song, A. E. Vasdekis, Z. Li, and D. Psaltis, Appl. Phys. Lett. 94, 161110 (2009).

${ }^{8}$ W. Song, A. E. Vasdekis, Z. Li, and D. Psaltis, Appl. Phys. Lett. 94, 051117 (2009).

${ }^{9}$ Z. Li and D. Psaltis, IEEE J. Sel. Top. Quantum Electron. 13, 185 (2007)

${ }^{10}$ M. Gersborg-Hansen and A. Kristensena, Appl. Phys. Lett. 89, 103518 (2006)

${ }^{11}$ Z. Li, Z. Zhang, A. Scherer, and D. Psaltis, Opt. Express 14, 10494 (2006).

${ }^{12}$ W. Song, A. E. Vasdekis, J. Choi, and D. Psaltis, CLEO, OSA, Paper CTuD2 (2009).

${ }^{13}$ Y. Sidorin and D. Howe, Opt. Lett. 22, 802 (1997).

${ }^{14}$ X. M. Zhang, A. Q. Liu, D. Y. Tang, and C. Lu, Appl. Phys. Lett. 84, 329 (2004).

${ }^{15}$ M. A. Unger, H. P. Chou, T. Thorsen, A. Scherer, and S. R. Quake, Science 288, 113 (2000). 